\title{
On the Use of Precession Electron Diffraction for Minerals Characterization: From Twinning Identification to Structure Refinement
}

\author{
Damien Jacob
}

\section{UMET, UMR 8207 CNRS-Université Lille 1, Villeneuve d'Ascq, France}

The precession electron diffraction (PED) technique [1] has been originally developed for structure determination at a submicrometer scale in a transmission electron microscope (TEM). Since, many structures have been solved using PED, recently combined with the tomographic acquisition of 3D electron diffraction data [2]. Using PED, integrated intensities of the diffracted beams as a function of the rocking beam orientation are collected. The resulting intensities keep dynamical in nature, due to residual multiple scattering, but are more closely related to the strength of the scattering events and ranking of reflections as a function of their intensities is generally correlated to the structure factor values, which is crucial for structure solution and refinement [3].

In the field of mineralogy, the sensitivity of PED intensities to small variations of the electron structure factors is of great use for microstructural characterizations. Furthermore, the technique suits well to beam sensitive materials since selected-area electron diffraction (SAED) patterns obtained using a defocus beam can be used. As an illustration, we show an example of twinning identification in coesite, a beam-sensitive high-pressure quartz polymorph [4]. In this case, lattices of the twinned individuals are nearly superimposed (pseudo-merohedral twinning) and conventional analysis using the orientation of the diffraction patterns is useless: only the reflection intensities as obtained using PED relate the symmetry operations between the twinned individuals (Figure 1). For this kind of applications, kinematical calculations of the reflection intensities are generally sufficient and the analysis is very fast and robust.

Using a more quantitative analysis of collected data, PED can be used for structure solution and refinement. In the last case, experimental intensities have to be compared with dynamical simulations of diffracted intensities, taking into account the multiple scattering events occurring when the electron beam is passing through the crystal. The analysis can be used to refine atomic occupancies of specific sites of the structure with mixed occupancies, giving access to ordering parameters. The described study concerns a natural orthopyroxene $\left(\mathrm{Mg}_{0.60} \mathrm{Fe}_{1.40}\right) \mathrm{Si}_{2} \mathrm{O}_{6}$ from metamorphic origin [5]. Samples of the same origin have also been heat-treated and water-quenched to maintain a disordered sate. In both cases (natural and heat-treated samples), the dynamical refinement of experimental diffracted intensities enables the estimation of the molar fractions $\mathrm{X}_{\mathrm{Fe}}(\mathrm{M} 1)$ and $\mathrm{X}_{\mathrm{Fe}}(\mathrm{M} 2)$ of Fe on $\mathrm{M} 1$ and $\mathrm{M} 2$ sites of the orthopyroxene structure. The measured values are closed to those deduced from XRD experiments at the grain scale (Figure 2). We will discuss on the accuracy of the method and on its potential application at a very local scale in the field of geospeedometry using terrestrial or extra-terrestrial material.

[1] R. Vincent and P.A. Midgley, Ultramicroscopy 53 (1994) p. 271.

[2] U. Kolb et al., Crystal Research and Technology 46 (2011), p. 542.

[3] W. Sinkler and L.D. Marks, Zeitschrift Fur Kristallographie 225 (2010) p. 47.

[4] D. Jacob et al., American Mineralogist 94 (2009) p. 684.

[5] D. Jacob et al., American Mineralogist 98 (2013) p.152. 

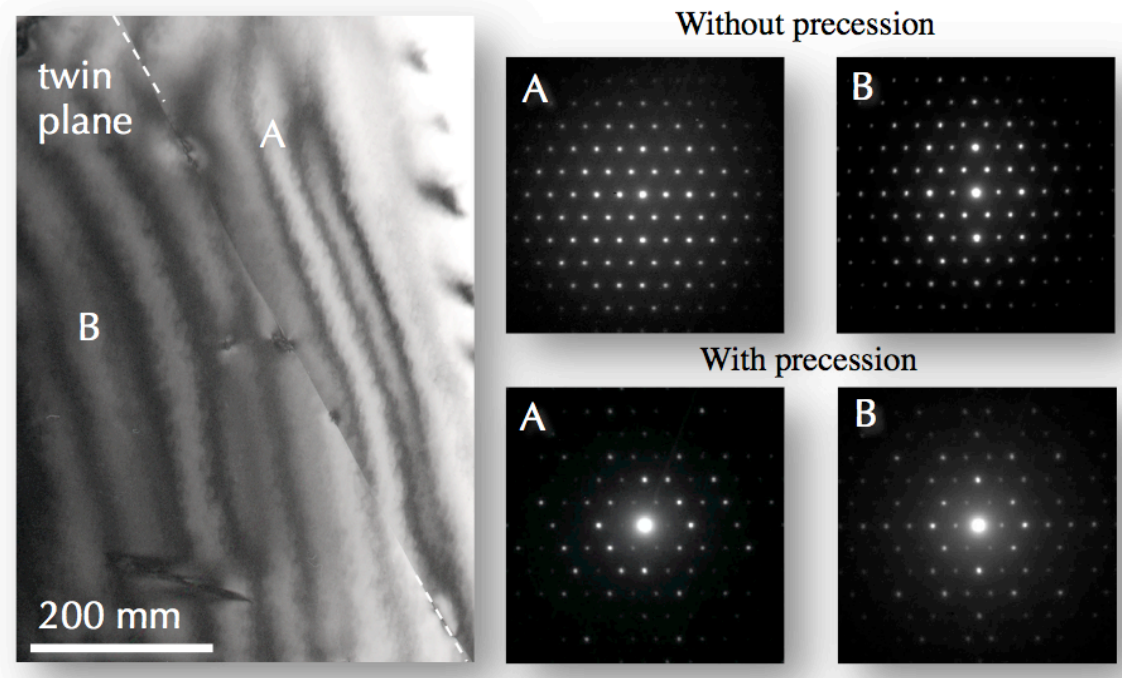

Figure 1. Bright-field image of a pseudo-merohedral twin in coesite together with the SAED patterns from both parts $\mathrm{A}$ and $\mathrm{B}$ of the twin. Without precession the pattern oreintation are not distinguishable. Using precession, diffracted intensities from both individuals reveal the twin law.

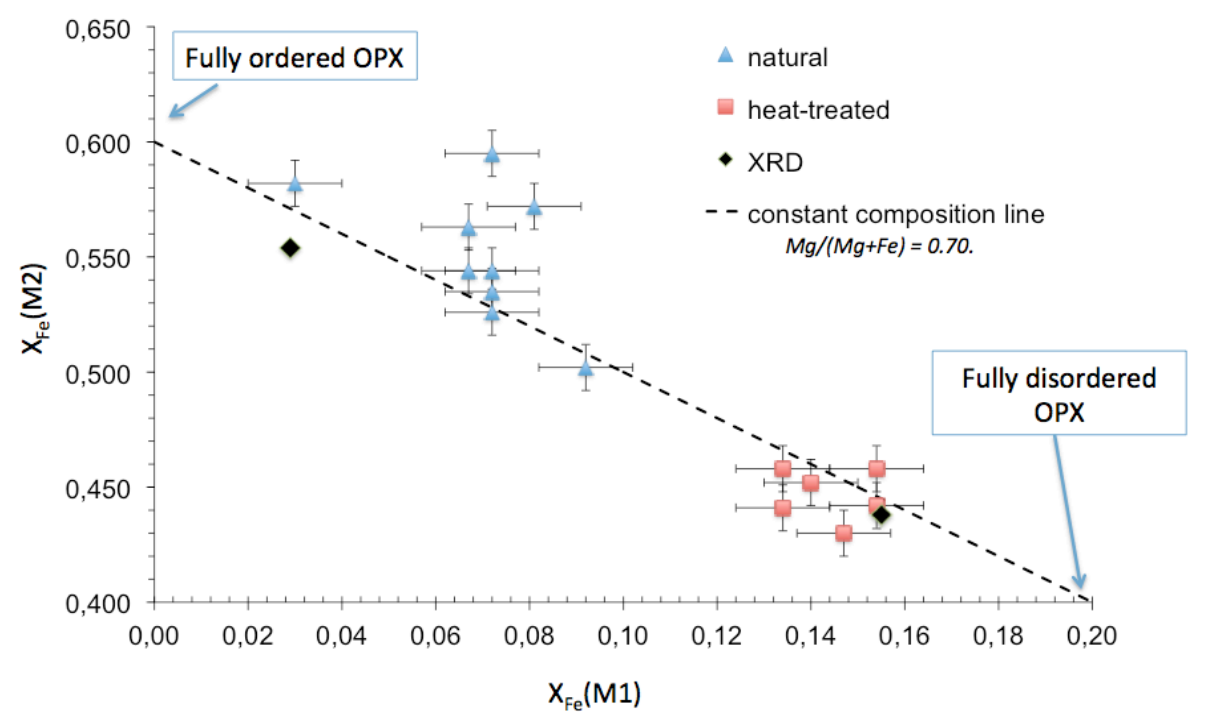

Figure 2. Plot of $\mathrm{X}_{\mathrm{Fe}}(\mathrm{M} 2)$ vs. $\mathrm{X}_{\mathrm{Fe}}(\mathrm{M} 1)$ in a natural and a heat-treated OPX samples from terrestrial origin as obtained from PED dynamical refinement. Results are in good agreement with those obtained form XRD data at the grain scale. 